\title{
A Strategy for Selecting Synchronization Sequences to Test Concurrent Object-Oriented Software ${ }^{1,2}$
}

\author{
Huo Yan Chen \\ Department of Computer Science \\ Jinan University, China \\ tchy@jnu.edu.cn
}

\author{
Yu Xia Sun \\ Department of Computer Science \\ Jinan University, China \\ sabrinna@,21cn.com
}

\author{
T. H. Tse $e^{3}$ \\ Department of Computer Science \\ and Information Systems \\ The University of Hong Kong \\ tse@csis.hku.hk
}

\begin{abstract}
Testing is the most commonly used approach to the assurance of software quality and reliability. The testing of object-oriented software is much more complex than that of conventional programs. Although we proposed previously a method called TACCLE for testing objectoriented software at the class and cluster levels, it did not cover concurrent or non-deterministic situations.

This paper puts forward a strategy for selecting synchronization sequences to test concurrent objectoriented software, including non-deterministic cases. It is based on OBJSA net/CLOWN specifications. Experiments have been carried out in a case study to verify the efficacy of the strategy.
\end{abstract}

Keywords: Object-oriented program testing, concurrency, non-determinism, OBJSA net

\section{Introduction}

Object-oriented programming is among the most popular software development technique today. It consists

\footnotetext{
${ }^{1}$ C 2003 IEEE. This material is presented to ensure timely dissemination of scholarly and technical work. Personal use of this material is permitted. Copyright and all rights therein are retained by authors or by other copyright holders. All persons copying this information are expected to adhere to the terms and constraints invoked by each author's copyright. In most cases, these works may not be reposted without the explicit permission of the copyright holder. Permission to reprint/ republish this material for advertising or promotional purposes or for creating new collective works for resale or redistribution to servers or lists, or to reuse any copyrighted component of this work in other works must be obtained from the IEEE.

${ }^{2}$ This research is supported by the National Natural Science Foundation of China under Grant \#60173038, the Guangdong Province Science Foundation under Grant \#010421, the Research Grants Council of Hong Kong under Grant No. HKU 7029/01E, and The University of Hong Kong under a CRCG grant.

${ }^{3}$ Corresponding author.
}

of many distinct features within the same paradigm. The application domain is modeled by objects that include not only data structures but all the operations also. Data abstraction and encapsulation allows us to separate object behaviors and interfaces from implementation details. Inheritance enhances reuse. Polymorphism enables us to use the same operation symbols for different purposes in different situations. The resulting software has been found to be more flexible, maintainable, and reusable.

On the other hand, objects may interact with one another in unforeseen combinations in object-oriented systems, which are much more complex to test than the hierarchical structures in conventional programs. Various proposals for testing object-oriented software system have been made [1-5]. In [1], for instance, we recommended a TACCLE methodology for testing object-oriented software at the class and cluster levels. We must concede, however, that it did not take concurrency and non-determinism into account.

Since the introduction of the Java language with strong multi-thread mechanisms and Internet support, the static analysis and dynamic testing of concurrency and non-determinism are increasing in importance. Although Carver and Tai [6] presented an effective technique for the use of sequencing constraints for specification-based testing of concurrent programs, these constraints are only limited to preceding and succeeding events. Although Zhu and $\mathrm{He}$ [7] put forward adequacy criteria for testing concurrent systems based on high-level Petri nets and proved subsumption relationships among them, the authors did not offer techniques for actually constructing test cases.

In [8], Chen presented an approach for the static analysis of concurrent and non-deterministic objectoriented programs in Java. As a supplement, we shall present in this paper a strategy for selecting synchronization sequences to dynamically test non-deterministic concurrent object-oriented software. The strategy is based on OBJSA net/CLOWN specifications $[9,10]$. In a 
companion paper [11], we shall further supplement this strategy by proposing a scheme for dynamically executing selected pairs of synchronization sequences.

An OBJSA net in CLOWN is composed of a superposed automata (SA) net inscribed with algebraic terms of an OBJ module. It supports the components-based analysis and incremental development of specifications with good modularity and reusability. By adding OBJ notions to Petri nets, such as order-sorted algebra, theory, and view [12], OBJSA net provides testers with more information for the selection of test cases. An OBJSA Netsupport Environment (ONE) has been built to facilitate construction and validation of the specifications.

We shall outline in Section 2 the underlying concepts and rules of OBJSA net specifications. Based on these fundamentals, we shall propose in Section 3 a strategy for selecting synchronization sequences to test concurrent non-deterministic object-oriented software. Section 4 presents an experimental case study to verify the effectiveness of the strategy. Section 5 concludes the paper.

\section{Underlying Concepts of OBJSA Nets}

In this section, we outline the underlying concepts of OBJSA nets, which were due originally to Battiston et al. Interested readers may refer to $[9,10]$ for more details.

\section{(a) Nets}

A net is a bipartite graph with two types of nodes: places and transitions. Places are used to model the statuses of system conditions and transitions are used to model operations that affect such statuses. More formally, a $n e t$ is a triple $(P, T, F)$ such that:

(i) $P$ is a finite non-empty set of places,

(ii) $T$ is a finite non-empty set of transitions,

(iii) $P \cap T=\varnothing$

(iv) $F \subseteq(P \times T) \cup(T \times P)$ is a set of arcs.

$V=P \cup T$ is known as the set of vertices. For any vertex $v \in V$, the set of $\operatorname{arcs}{ }^{\circ} v=\{x \in V \mid(x, v) \in F\}$ is called its pre-set, and the set of $\operatorname{arcs} v^{o}=\{y \in V \mid(v, y) \in F\}$ is called its post-set.

\section{(b) Extended SA Nets}

Given a net $(P, T, F)$, an extended $S A$ net $N$ is a tuple $(P, T, F, W, \Pi)$ satisfying the following conditions:

(i) $O P$ is a set of open places and $C P$ is a set of closed places such that $O P \cup C P=P$ and $O P \cap C P=\varnothing$.

(ii) OT is a set of open transitions and $C T$ is a set of closed transitions such that $O T \cup C T=T$ and $O T \cap C T=\varnothing$. (iii) $O F \subseteq(O P \times O T) \cup(O T \times O P)$ is a set of open arcs.

(iv) $C F \subseteq(C P \times T) \cup(T \times C P)$ is a set of closed arcs.

(v) $W: F \rightarrow N a t$ is an arc weight function. It assigns to every arc a natural number denoting its weight. In particular, every open arc is assigned a weight of 1 .

(vi) $\Pi$ is a partition of $P$, dividing it into $m$ subsets $\left(\prod_{i}\right)_{i=1,2, \ldots, m}$ such that $\prod_{1} \cup \Pi_{2} \cup \ldots \cup \prod_{m}=P$ and $\prod_{i} \cap \prod_{j}=\varnothing$ for $i \neq j$. Each $\prod_{i}$ contains open places only or closed places only. For every transition $t \in T$, the total weights of all the arcs from the places in $\prod_{i}$ to $t$ is the same as the total weights of all the arcs from $t$ to the places in $\prod_{i}$.

An extended SA net is open if it contains at least one open place or open transition; otherwise it is closed. An extended SA net is elementary if it is contains only closed places.

\section{(c) OBJSA Nets}

Let $N=(P, T, F, W, \Pi)$ be an extended SA net and $S P E C=(S, \Sigma, E)$ be an algebraic specification, where $S$ is a set of sorts (or object classes), $\Sigma$ is a family of operation symbols, and $E$ is a set of equational axioms, possibly conditional. An OBJSA component is a SPEC-inscribed net $(N$, ins, SPEC), where ins $=(\varphi, \lambda, \eta)$ satisfies the following conditions:

(i) $\varphi: P \rightarrow S$ is a sort assignment function. It classifies the places into different sorts while respecting the partition $\Pi$. Every element of sort $\varphi(p)$ is called a token. It is of the form $\langle n, d\rangle$, where $n$ denotes the token name and $d$ denotes the data content.

(ii) $\lambda: F \rightarrow \operatorname{Term}_{S, \Sigma}[X]$ is an arc labeling function. It assigns labels to the arcs while respecting the sort assignment. Given any transition $t \in T$ and any place $p \in{ }^{\circ} t$, the label of the $\operatorname{arc} f=(p, t)$ is the concatenated string $x_{1} \oplus x_{2} \oplus \ldots \oplus x_{W(f)}$. Each $x_{i}$ is a variable of sort $\varphi(p)$. Let $X_{t}$ be a list of variables that label the input $\operatorname{arcs}$ of $t$, and $X$ be the set of variables for all the arcs of the net. Given any transition $t \in T$ and any place $q \in$ $t^{\circ}$, the label of the $\operatorname{arc} f=(t, q)$ is the concatenated string $y_{1}\left(X_{t}\right) \oplus y_{2}\left(X_{t}\right) \oplus \ldots \oplus y_{W(f)}\left(X_{t}\right)$. Each $y_{j}\left(X_{t}\right)$ is a term of the form $\left\langle n_{j}^{\prime}, d_{j}^{\prime}\right\rangle$ of sort $\varphi(q)$ such that $n_{j}^{\prime}=n_{i}$ and $d_{j}^{\prime}=\sigma_{t}\left(\ldots, d_{i}, \ldots\right)$ for some $x_{i}=\left\langle n_{i}, d_{i}\right\rangle$ in $X_{t}$ and for some operation $\sigma_{t}$ that specifies the change of the data content due to the transition $t$.

(iii) $\eta: T \rightarrow 2^{X} \rightarrow$ Bool is a guard function that assigns a pre-condition $\eta(t)\left(X_{t}\right)$ to every transaction $t$ before it can be fired.

Furthermore, an OBJSA component is associated with an initial marking (or initial state) $M_{0}$, which assigns to each closed place $p$ a family of tokens of sort $\varphi(p)$. 
An OBJSA component is elementary if the underlying net $N$ contains only an elementary subnet. An OBJSA component is open if it is formed by composing elementary or other open components, such that the underlying net is open. Finally, an OBJSA net is a closed OBJSA component, formed by composing elementary or open OBJSA components, such that the underlying net is closed.

\section{(d) Firing Mode}

Given an OBJSA component, a firing mode for a transition $t \in T$ is an assignment function $\beta_{t}: X_{t} \rightarrow$ Term $_{S, \Sigma}$ that substitutes every variable $x \in X_{t}$ by a ground term (that is, a term without variables) of sort $\varphi(p)$ some $p \in{ }^{\circ} t$.

For a place $\mathrm{p} \in P$, a transition $t \in T$, and a firing mode $\beta_{t}$, if $p \in{ }^{\circ} t$, then $\operatorname{IN}(p, t)$ is defined as $\left\{\beta_{t}\left(x_{i}\right) \mid i=\right.$ $1,2, \ldots, W(p, t)\}$; otherwise $I N(p, t)$ is defined as the empty set. Similarly, if $p \in t^{0}$, then $\operatorname{OUT}(t, p)$ is defined as $\left\{\beta_{t}\left(y_{j}\left(X_{t}\right)\right) \mid j=1,2, \ldots, W(t, p)\right\}$; otherwise $\operatorname{OUT}(t, p)$ is defined as the empty set. Given a marking $M$, a transition $t \in T$, and a firing mode $\beta_{t}$, if $\eta(t)\left(\beta_{t}\left(X_{t}\right)\right)=$ true and $I N(p, t)$ $\subseteq M(p)$ for every place $p \in{ }^{\circ} t$, then $t$ is said to be $\beta_{t}$-enabled at $M$. In this case, $t$ may fire in mode $\beta_{t}$. Such firing returns a new marking $M^{\prime}$ such that $M^{\prime}(p)=M(p) \backslash I N(p, t)$ $\cup \operatorname{OUT}(t, p)$ for every $p \in P$.

\section{Our Strategy for Selecting Synchronization Sequences}

Based on the concept of OBJSA nets, we present in this section a strategy for selecting synchronization sequences to test non-deterministic concurrent objectoriented software.

Given an OBJSA net $O s n$, we say that a marking $M$ is reachable if, starting from the initial marking $M_{0}$, we can obtain $M$ by firing a sequence $\tau$ of consecutively enabled transitions. In this case, we call $\tau$ an enabled sequence and write $M_{0} \stackrel{\tau}{\longrightarrow} M$. We assume that $\tau$ is annotated with arc information so that any intermediate marking can be tracked unambiguously. If $\tau=n u l l, M=M_{0}$.

Let $M^{*}=\left\{M \mid M_{0} \stackrel{\tau}{\longrightarrow} M\right.$ for some enabled sequence $\tau\}$ be the set of reachable markings of Osn. For any $M \in M^{*}$, the number of non-deterministic enabled transitions of $O s n$ at $M$ is called the enabled degree of Osn at $M$, and is denoted by $e d(O s n, M)$. Let $m d(O s n)=$ $\max \left\{e d(\right.$ Osn, $\left.M) \quad \mid \quad M \in M^{*}\right\} \quad$ be the maximum non-deterministic enabled degree. We say that $M_{g}$ is a goal marking of Osn if $e d\left(O s n, M_{g}\right)=m d(O s n)$. Let $E T\left(M_{g}\right)$ be the set of enabled transitions at $M_{g}$. Obviously, $\left|\operatorname{ET}\left(M_{g}\right)\right|$ $\geq m d(O s n)$. $m d(O s n)$ must exist, because $e d(O s n, M)$ for any $M \in$ $M^{*}$ must be a non-negative integer satisfying $\operatorname{ed}(\operatorname{Osn}, M) \leq$ $|T|$. The goal marking $M_{g}$ corresponding to $m d(O s n)$, however, is not necessarily unique. At $M_{g}$, the firings of transitions in $O s n$ have the maximum non-determinism and hence the maximum competition on system resources. At this moment, the system is in a most complex state and is therefore most error prone. Hence, we should catch the state corresponding to $M_{g}$ in our testing. This is the motivation and the general idea of our strategy for selecting synchronization sequences.

$$
\text { Suppose } M_{0} \stackrel{\tau_{g}}{\longrightarrow} M_{g} \text {. Let } T C_{g}=\left\{\tau_{g} \cdot t_{i} \mid\right.
$$
$\left.t_{i} \in E T\left(M_{g}\right)\right\}$, where $\tau_{g} \cdot t_{i}$ denotes the firing of $t_{i}$ immediately after $\tau_{g}$. We say that $\tau_{g}$ is a goal sequence and $T C_{g}$ is a goal set. Our strategy is to select $T C_{g}$ as a set of initial test cases.

Let $T C$ be any set of test cases. For any given transition $t \in T$, is there an $M \in M^{*}$ such that $t$ is enabled at $M$ ? The problem is difficult because $M^{*}$ is infinite in general, and is NP-hard even if $\mathrm{M}^{*}$ is finite.

From the complexity point of view, seeking a goal marking $M_{g}$, goal sequence $\tau_{g}$, or a goal set $T C_{g}$ is also a difficult problem. For a particular $O s n$, however, we can give a heuristic strategy for seeking a ballpark goal marking $M_{g}{ }^{\prime}$ and the corresponding ballpark goal sequence $\tau_{g}{ }^{\prime}$ such that there will be as many enabled transitions at $M_{g}{ }^{\prime}$ as possible. The corresponding ballpark goal set $T C_{g}{ }^{\prime}$ is $\left\{\tau_{g}{ }^{\prime} \cdot t_{i} \mid t_{i} \in E T\left(M_{g}{ }^{\prime}\right)\right\}$.

An enabled transition at initial marking $M_{0}$ is called a source transition of Osn. The number of times that a source transition in an enabled sequence can be consecutively fired from $M_{0}$ is known as the index of the source transition. We shall refer to a source transition with the largest index as the greatest index source transition, or GIST for short.

Suppose $t_{0}$ is the GIST of $O s n$ with $M_{0}$. Our heuristic strategy to seek a ballpark goal marking $M_{g}{ }^{\prime}$ and the corresponding ballpark goal sequence $\tau_{g}^{\prime}$ includes a reachability search [13-17], such as the following hill-climbing procedure:

(1) set $\tau_{g}{ }^{\prime}=$ null and $M_{g}{ }^{\prime}=M_{0}$;

(2) fire $t_{0}$ at $M_{0}$ and obtain $M_{1}$, that is, $M_{0} \stackrel{t_{0}}{\longrightarrow} M_{1}$;

(3) at $M_{1}$ :

if $t_{0}$ is not enabled, return $\tau_{g}{ }^{\prime}$ and $M_{g}{ }^{\prime}$, and exit;

if $t_{0}$ is still enabled, \{

if there is no other enabled transition $t_{1}$, return $\tau_{g}{ }^{\prime}$ and $M_{g}{ }^{\prime}$, and exit;

if there is another enabled transition $t_{1},\{$ 


$$
\begin{aligned}
& \text { set } \tau_{g}{ }^{\prime}=\tau_{g}{ }^{\prime} \cdot t_{0}, M_{1}{ }^{\prime}=M_{1}, M_{g}{ }^{\prime}=M_{1} \text {, and } i=1 \text {; } \\
& \} \text {; }
\end{aligned}
$$

(4) if there are adequately many ${ }^{4}$ non-deterministic enabled transitions at $M_{g}{ }^{\prime}$, then return $\tau_{g}{ }^{\prime}$ and $M_{g}{ }^{\prime}$, and exit; otherwise $M_{i}^{\prime} \stackrel{{ }_{i}}{\longrightarrow} M_{i+1}$;

(5) at $M_{i+1}$ :

if $t_{0}$ is not enabled, return $\tau_{g}{ }^{\prime}$ and $M_{g}{ }^{\prime}$, and exit; if $t_{0}, t_{1}, \ldots, t_{i}$ are still enabled, \{

if there is no other enabled transition $t_{i+1}$, return $\tau_{g}{ }^{\prime}$ and $M_{g}{ }^{\prime}$, and exit;

if there is another enabled transition $t_{i+1},\{$ set $\tau_{g}{ }^{\prime}=\tau_{g}{ }^{\prime} \cdot t_{i}, M_{i+1}{ }^{\prime}=M_{i+1}, M_{g}{ }^{\prime}=M_{i+1}{ }^{\prime}$, and $i=i+1$

go to (4);

\} ;

\} ;

if $t_{0}$ is still enabled but some of $t_{1}, t_{2}, \ldots, t_{i}$ are not enabled, \{

if firing some of $t_{0}, t_{1}, \ldots, t_{i-1}$ several times can enable all of $t_{0}, t_{1}, \ldots, t_{i},\{$

let $\tau_{i}$ be the corresponding sequence of the fired transitions and let $M_{i+1}{ }^{\prime}$ be the marking

obtained, that is, $M_{i+1} \stackrel{\tau_{i}}{\longrightarrow} M_{i+1}^{\prime}$;

if there is no other enabled transition $t_{i+1}$ at $M_{i+1}{ }^{\prime}$, return $\tau_{g}{ }^{\prime}$ and $M_{g}{ }^{\prime}$, and exit;

if there is another enabled transition $t_{i+1}$ at $M_{i+1}{ }^{\prime},\{$ set $\tau_{g}{ }^{\prime}=\tau_{g}{ }^{\prime} \cdot t_{i} \cdot \tau_{i}, M_{g}{ }^{\prime}=M_{i+1}{ }^{\prime}$, and $i=i+1$; go to (4); \} ;

\}

else return $\tau_{g}{ }^{\prime}$ and $M_{g}{ }^{\prime}$, and exit;

\} ;

In order to understand the strategy, readers may like to construct a decision tree for the above steps.

After obtaining the ballpark goal sequence $\tau_{g}{ }^{\prime}$ and the corresponding $M_{g}{ }^{\prime}$, we can construct the ballpark goal set $T C_{g}{ }^{\prime}=\left\{\tau_{g}{ }^{\prime} \cdot t_{i} \mid t_{i} \in E T\left(M_{g}{ }^{\prime}\right)\right\}$ and take $T C_{g}{ }^{\prime}$ as a set of test cases.

A transition in OBJSA net represents an operation in the implementation triggered by some message passing from another object. A test case in $T C_{g}{ }^{\prime}$ represents a sequence of inter-object operations in the implementation, which we shall call a synchronization sequence. We can then substitute the relevant parts of these synchronization

\footnotetext{
${ }^{4}$ That is, in the most recent $m$ loops, the number of enabled transitions at $M_{g}{ }^{\prime}$ does not increase, where $m$ is a parameter specified by the user.
}

sequences into the axioms of corresponding classes, and apply our class-level testing strategy for observational equivalence [1]. Because of the non-determinism of concurrent programs, we must use a replay technique to execute each synchronization sequence in $T C_{g}{ }^{\prime}$. Details of replay techniques can be found, for example, in [18].

Note that $T C_{g}{ }^{\prime}$ may not cover all the transitions of $O s n$. In this case, and if $T C_{g}{ }^{\prime}$ cannot reveal errors, we also need to construct other sets of test cases covering the remaining transitions $T \backslash\left(E T\left(M_{g}{ }^{\prime}\right) \cup\left\{t \mid t\right.\right.$ appears in $\left.\left.\tau_{g}{ }^{\prime}\right\}\right)$ as supplements. Finally, the testing of Osn may be repeated by choosing other initial markings. Such initial markings may be given in the specification, or obtained from a reachability search or category-partitioning scheme.

\section{Case Study and Experiments}

As a case study, we have applied the above strategy to a system consisting of four generators and five users synchronously exchanging messages through four buffers. These three constituents can be specified by OBJSA open components Generator, User, and Buffer, respectively. Each object is identified by a natural number together with the component type. The structures of these components are similar to those in the worked example in [9]. The OBJSA net specifying the integrated system fused from these components is shown in Figure 1. Suppose an initial marking $M_{0}$ is as follows:

$$
\begin{gathered}
M_{0}(g 2)=\{\langle[g, 1], \text { null }\rangle,\langle[g, 2], \text { null }\rangle, \\
\langle[g, 3], \text { null }\rangle,\langle[g, 4], \text { null }\rangle\} ; \\
M_{0}(u 2)=\{\langle[u, 1], \text { nullms }\rangle,\langle[u, 2], \text { nullms }\rangle, \\
\langle[u, 3], \text { nullms }\rangle,\langle[u, 4], \text { nullms }\rangle, \\
\langle[u, 5], \text { nullms }\rangle\} ; \\
M_{0}(b 1)=\{\langle[b, 1], \text { nullms }\rangle\rangle,\langle[b, 2], \text { nullms }\rangle, \\
\langle[b, 3], \text { nullms }\rangle\rangle,\langle[b, 4], \text { nullms }\rangle\} ; \\
M_{0}(p)=\varnothing \text { for } p \in P \backslash\{g 2, u 2, b 1\} .
\end{gathered}
$$

Following the definition in Section 4, we obtain the GIST $t_{0}=$ generate. Following the proposed strategy, we have the following process: (i) Set $\tau_{g}{ }^{\prime}=$ null and $M_{g}{ }^{\prime}=M_{0}$. (ii) Fire $t_{0}$ and obtain $M_{1}$ by the rules in Section 2. (iii) Here $t_{0}$ is still enabled. By the definition in Section 2, another transition $t_{1}=$ fetch is enabled. Set $\tau_{g}{ }^{\prime}=$ generate, $M_{1}{ }^{\prime}=M_{1}$, $M_{g}{ }^{\prime}=M_{1}$, and $i=1$. (iv) Fire $t_{1}=$ fetch and obtain $M_{2}$ by the rules in Section 2. (v) Here $t_{0}$ is still enabled but $t_{1}$ is not. Fire $\tau_{1}=t_{0}$ and obtain $M_{2}^{\prime}$. By the rules in Section 2, another transition $t_{2}=$ accept is enabled. Set $\tau_{g}{ }^{\prime}=$ generate $\cdot$ fetch $\cdot$ generate, $M_{g}{ }^{\prime}=M_{2}{ }^{\prime}$, and $i=2$. Go back to step (4) of the strategy. 


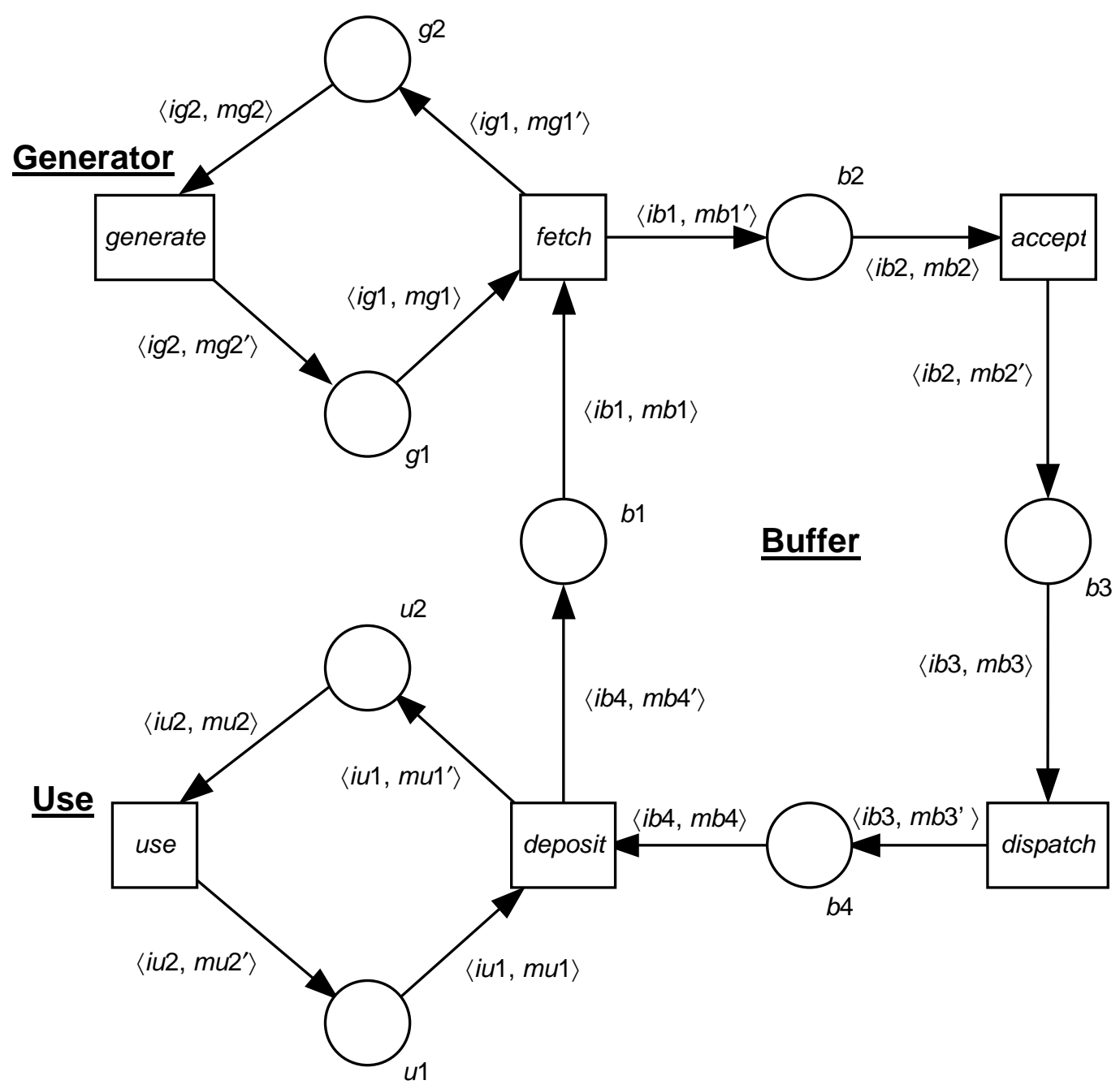

$i g 1$, ig2, iu1, iu2, ib1, ib2, ib3, ib4: ObjectName in the form [type: Type, id: Nat], where Type denotes the set of object sorts, Nat denotes the set of natural numbers, type $\in\{g, u, b\}, g$ denotes generator, $u$ denotes user, $b$ denotes buffer, and id denotes object identity number.

$m g 1, m g 2, m g 1^{\prime}, m g 2^{\prime}$, null: Message. $\quad m g 1^{\prime}=$ null. $\quad m g 2^{\prime}=$ generateMessage(ig2).

$m u 1, m u 2, m u 1^{\prime}, m u 2^{\prime}$, nullmsg: FullMessage in the form [msg: Message, orig: Nat], where orig denotes the originating object. $\quad m u 1^{\prime}=m b 4 . \quad m u 2^{\prime}=$ nullmsg.

$m b 1, m b 2, m b 3, m b 4, m b 1^{\prime}, m b 2^{\prime}, m b 3^{\prime}, m b 4^{\prime}:$ FullMessage. $\quad m b 1^{\prime}=[m g 1, n u m(i g 1)] . \quad m b 2^{\prime}=m b 2 . \quad m b 3^{\prime}=m b 3$. $m b 4^{\prime}=$ nullmsg.

Figure 1. Case Study 
Finally, we have obtained the ballpark goal sequence $\tau_{g}{ }^{\prime}=$ generate $\cdot$ fetch $\cdot$ generate $\cdot$ accept $\cdot$ generate $\cdot$ fetch $\cdot$ dispatch $\cdot$ generate $\cdot$ fetch $\cdot$ accept $\cdot$ deposit $\cdot$ generate fetch $\cdot$ accept $\cdot$ dispatch. Obviously, in this case we have $E T\left(M_{g}{ }^{\prime}\right)=T$, and hence the ballpark goal set $T C_{g}{ }^{\prime}$ is $\left\{\tau_{g}{ }^{\prime} \cdot\right.$ generate, $\tau_{g}{ }^{\prime} \cdot$ fetch,$\tau_{g}{ }^{\prime} \cdot$ accept,$\tau_{g}{ }^{\prime} \cdot$ dispatch, $\tau_{g}{ }^{\prime} \cdot$ deposit, $\tau_{g}{ }^{\prime} \cdot$ use $\}$. This can then be followed-up with class-level testing of observational equivalence [1].

We have implemented, in Java, a system consisting of these components. We have embedded 30 different mutants into the program. The above strategy in generating the test set $T C_{g}{ }^{\prime}$ can kill all the mutants.

\section{Conclusion}

Based on an OBJSA net/CLOWN specification, we have proposed a strategy to select synchronization sequences for testing non-deterministic concurrent objectoriented software. We have reported an effectiveness case study and experiments on the proposed strategy. More case studies and experiments are being planned.

\section{Acknowledgement}

We are grateful to Shuang Quan Li of Jinan University for implementing the system and conducting the experiments and tests in the case study.

\section{References}

[1] H. Y. Chen, T. H. Tse, and T. Y. Chen. TACCLE: a methodology for object-oriented software testing at the class and cluster levels. ACM Transactions on Software Engineering and Methodology, 10 (1): 56-109, 2001.

[2] R.-K. Doong and P. G. Frankl. The ASTOOT approach to testing object-oriented programs. ACM Transactions on Software Engineering and Methodology, 3 (2): 101-130, 1994.

[3] D. C. Kung, J. Z. Gao, P. Hsia, Y. Toyoshima, and C. Chen. A test strategy for object-oriented programs. In Proceedings of the 19th Annual International Computer Software and Applications Conference (COMPSAC '95), pages 239-244. IEEE Computer Society Press, Los Alamitos, California, 1995.

[4] M. D. Smith and D. J. Robson. A framework for testing object-oriented programs. Journal of Object-Oriented Programming, 5 (3): 45- 53, 1992.

[5] C. D. Turner and D. J. Robson. A state-based approach to the testing of class-based programs. Software: Concepts and Tools, 16 (3): 106-112, 1995.

[6] R. H. Carver and K.-C. Tai. Use of sequencing constraints for specification-based testing of concurrent programs. IEEE Transactions on Software Engineering, 24 (6): 471490, 1998.
[7] H. Zhu and X. He. A theory of testing high-level Petri nets. In Proceedings of the International Conference on Software: Theory and Practice, 16th IFIP World Computer Congress, pages 443-450. Beijing, China, 2000.

[8] H. Y. Chen. Race condition and concurrency safety of multithreaded object-oriented programming in Java. In Proceedings of the 2002 IEEE International Conference on Systems, Man, and Cybernetics (SMC 2002), pages 134-139. IEEE Computer Society Press, Los Alamitos, California, 2002 .

[9] E. Battiston, F. de Cindio, and G. Mauri. Modular algebraic nets to specify concurrent systems. IEEE Transactions on Software Engineering, 22 (10): 689-705, 1996.

[10] E. Battiston, A. Chizzoni, and F. D. Cindio. CLOWN as a testbed for concurrent object-oriented concepts. In Concurrent Object-Oriented Programming and Petri Nets: Advances in Petri Nets, pages 131-163. Lecture Notes on Computer Science, Springer, Berlin, 2001.

[11] H. Y. Chen, Y.X. Sun, and T. H. Tse, "A scheme for dynamic detection of concurrent execution of objectoriented software", in Proceedings of the 2003 IEEE International Conference on Systems, Man, and Cybernetics (SMC 2003), IEEE Computer Society Press, Los Alamitos, California, 2003.

[12] J. A. Goguen, T. Winkler, J. Meseguer, K. Futatsugi, and J.-P. Jouannaud. Introducing OBJ3. In J. A. Goguen and G. Malcolm, editors, Software Engineering with OBJ: Algebraic Specification in Action. Kluwer Academic Publishers, Boston, 2000.

[13] S. C. Cheung and J. Kramer. Context constraints for compositional reachability analysis. ACM Transactions on Software Engineering and Methodology, 5(4): 334-377, 1996.

[14] H. S. Hong, S. D. Cha, D. H. Bae, and H. Ural. A test sequence selection method for statecharts. Software Testing, Verification and Reliability, 10(4): 203-257, 2000.

[15] T. H. Kim, I. S. Hwang, M. S. Jang, and J. Y. Lee. Test case generation of a communication protocol by an adaptive state exploration. Computer Communications, 24(13): 1242$1255,2001$.

[16] D. Lee, K. K. Sabnani, D. M. Kristol, and S. Paul. Conformance testing of protocls specified as communicating finite state machines: a guided random walk based approach. IEEE Transactions on Communications, 44(5): 631-640, 1996.

[17] P. Tripathy and B. Sarikaya. Test generation from LOTOS specifications. IEEE Transactions on Computers, 40(4): 543-552, 1991.

[18] R. H. Carver and K.-C. Tai. Replay and testing for concurrent programs. IEEE Software, 8 (3): 66-74, 1991. 